\title{
Protein interface prediction using solvent accessibility of unbound residues
}

\author{
Jennifer Mortensen ${ }^{1}$, Thom Vreven ${ }^{1}$, and Zhiping Weng ${ }^{1}$ \\ ${ }^{1}$ University of Massachusetts Medical School
}

May 5, 2020

\begin{abstract}
The prediction of protein-protein interfaces requires both the identification of interface residues and the proper spatial orientation of the component proteins. Many methods have been developed to identify interface residues, often using relative interface propensity (RIP), the enrichment of a particular amino acid type at the interface compared to the rest of the protein surface. We aimed to improve RIP for interface identification by incorporating the solvent accessibility of each amino acid. We studied the surface residues of 290 unbound structures corresponding to components of protein complexes and compared the relative solvent accessible surface area (rSASA) distributions of residues that end up in the interface and those that do not. Our results show that the side-chains of amino acids that become interface residues are more solvent exposed than non-interface surface residues on the unbound protein structure. Using this knowledge, we created an rSASA-dependent probability of becoming an interface residue for each amino acid type. Our results show that the solvent accessible surface area of residues should be taken into account when identifying interface residues and can be applied to other interface prediction techniques that use RIP to improve their results.
\end{abstract}

\section{Hosted file}

Predicting Interfaces Mortensen.pdf available at https://authorea.com/users/316811/articles/ 446945-protein-interface-prediction-using-solvent-accessibility-of-unbound-residues 